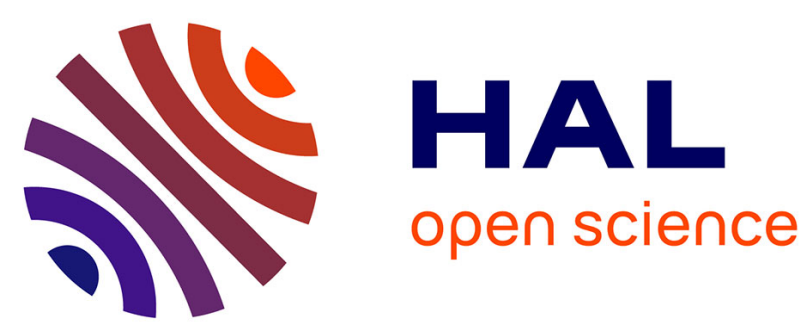

\title{
Time course of odorant- and trigeminal-induced activation in the human brain: an event-related functional magnetic resonance imaging study
}

P.E. Billot, A. Comte, E. Galliot, P. Andrieu, V. Bonnans, L. Tatu, T. Gharbi, T. Moulin, J.-L. Millot

\section{To cite this version:}

P.E. Billot, A. Comte, E. Galliot, P. Andrieu, V. Bonnans, et al.. Time course of odorant- and trigeminal-induced activation in the human brain: an event-related functional magnetic resonance imaging study. Neuroscience, 2011, 189, pp.370-376. 10.1016/j.neuroscience.2011.05.035 . hal00655049

\section{HAL Id: hal-00655049 \\ https://hal.science/hal-00655049}

Submitted on 30 Apr 2021

HAL is a multi-disciplinary open access archive for the deposit and dissemination of scientific research documents, whether they are published or not. The documents may come from teaching and research institutions in France or abroad, or from public or private research centers.
L'archive ouverte pluridisciplinaire HAL, est destinée au dépôt et à la diffusion de documents scientifiques de niveau recherche, publiés ou non, émanant des établissements d'enseignement et de recherche français ou étrangers, des laboratoires publics ou privés. 


\section{TIME COURSE OF ODORANT- AND TRIGEMINAL-INDUCED ACTIVATION IN THE HUMAN BRAIN: AN EVENT-RELATED FUNCTIONAL MAGNETIC RESONANCE IMAGING STUDY}

\author{
P.-E. BILLOT, ${ }^{a}$ A. COMTE, a,b E. GALLIOT, ${ }^{a}$ P. ANDRIEU, ${ }^{a}$ \\ V. BONNANS, ${ }^{b}$ L. TATU, ${ }^{a, c}$ T. GHARBI, ${ }^{d}$ \\ T. MOULIN ${ }^{\mathrm{a}, \mathrm{b}}$ AND J.-L. MILLOT ${ }^{\mathrm{a} *}$ \\ a Laboratoire de Neurosciences Intégratives et Cliniques, Université de \\ Franche-Comté, 2 Place Leclerc, 25030 Besançon Cedex, France \\ ${ }^{b}$ Département de Neuroimagerie Fonctionnelle, Centre d'Investigation \\ Clinique-Innovation Technologique, Centre Hospitalier Universitaire \\ J Minjoz, Boulevard Fleming, 25030 Besançon Cedex, France \\ 'Département d'Anatomie, Centre Hospitalier Universitaire J Minjoz, \\ Boulevard Fleming, 25030 Besançon Cedex, France \\ 'Laboratoire d'Electronique, Mécanique et Optique Unité Mixte de \\ Recherche CNRS 6174, Université de Franche-Comté, Route de \\ Gray, 25030 Besançon Cedex, France
}

\begin{abstract}
It is well known that most odorants stimulate the trigeminal system but the time course of the brain regions activated by these chemical stimulations remains poorly documented, especially regarding the trigeminal system. This functional magnetic resonance imaging (fMRI) study compares brain activations resulting from the contrast between two odorant conditions (one bimodal odor and one relatively pure olfactory stimulant) according to the duration of the stimulation (i.e. one inhalation, or three or six successive inhalations). The results show striking differences in the main brain regions activated according to these durations. The caudate nucleus and the orbitofrontal cortex are only involved in short-duration stimulations, and the posterior insular cortex and post-central gyrus (SI) are only activated by long duration stimulations. Different regions of the frontal, temporal and occipital lobe are activated depending on the duration but mainly during medium-duration stimulations. These results expand on the findings of previous studies and contribute to the description of temporal networks in trigeminal perception.
\end{abstract}

Key words: trigeminal, fMRI, olfaction, bimodal odor.

Most odorants are simultaneously perceived in humans by two sensory systems. They stimulate receptors of the olfactory epithelium, and thus the olfactory nerve (cranial nerve I), as well as free nerve endings and specific receptors in the nasal cavity, and thus the trigeminal nerve (cranial nerve V) (Doty et al., 1978; Hummel, 2000). Although the brain processes corresponding to their integration contribute to the various perceptions of odors and flavors (Albrecht et al., 2010), the interactions between these two sensory systems remain poorly understood.

${ }^{*}$ Corresponding author. Tel: +33-3-81-66-57-19; fax: +33-3-81-6657-46.

E-mail address: jean-louis.millot@univ-fcomte.fr (J.-L. Millot).

Abbreviations: AA, isoamyl acetate; PEA, phenyl ethyl alcohol.
Due to numerous studies in functional cerebral imaging, the brain areas affected by olfactory stimulation and perception are now well-known. Stimulation by any pure or relatively pure olfactory stimulant mainly activates primary olfactory regions (i.e. piriform cortex, amygdala and neighboring cortex) and secondary olfactory regions (hippocampus, orbitofrontal cortex and insula) (Zatorre et al., 1992; Royet et al., 2001; Gottfried et al., 2002; Savic, 2002). Asymmetry between the two hemispheres can be constant (e.g. the right orbitofrontal cortex is predominantly activated) or odor dependent (left amygdala appears to be more sensitive to unpleasant odors). Other brain areas may be involved depending on the subject's odor-related task: judgments of intensity, familiarity, memory or characteristics of hedonic valence (Royet et al., 2003). Nevertheless, studies on time course-induced activations have shown that some brain areas are successively affected by these processes. First and foremost, short bursts of stimulation activate the piriform cortex, hippocampus and part of the insula. These activities decrease in long duration stimulations which lead to a strong recruitment of the right orbitofrontal cortex (Sobel et al., 2000; Poellinger et al., 2001).

The literature on the functional neuroanatomy of trigeminal perception is less substantial. Contrasting or comparing brain activations due to odorants with different trigeminal properties is a common and fruitful approach to specifying the brain activations elicited by the trigeminal component of an odorant. Studies have shown additional activations of the insula, cingulum and cerebellum with bimodal (olfactory-trigeminal) stimuli compared to "pure" olfactory stimulants (Yousem et al., 1997; Bengtsson et al., 2001; Savic et al., 2002; Lombion et al., 2009). Hummel et al. (2005) and Boyle et al. (2007a,b) used $\mathrm{CO}_{2}$ (a relatively selective trigeminal nerve stimulant) as a referent and found additional activations in the midbrain, caudate nucleus, middle cingulate and temporal and frontal gyri. Iannilli et al. (2007) investigated brain activations in anosmic subjects in response to $\mathrm{CO}_{2}$ and mainly found activations in parts of the cerebellum and the temporal, parietal and frontal cortices. Nevertheless, the time course of activations for these areas involved in trigeminal perception remains largely unknown. Previous studies have used either relatively long durations, from $15 \mathrm{~s}$ to $30 \mathrm{~s}$ (Yousem et al., 1997; Bengtsson et al., 2001; Savic et al., 2002) or "puffs" of odors lasting $1 \mathrm{~s}$ or less (Boyle et al., 2007a,b; lannilli et al., 2007). The present study aimed to define some of the elements of the time-dependent processes in response to trigeminal stimulation. As in a previous study (Lombion et 
al., 2009) we used phenyl ethyl alcohol (PEA), a rose-like odor, and isoamyl acetate (AA), a banana-like odor as odorants. PEA is a relatively pure olfactory stimulus and AA is a bimodal stimulus; they are detected by one of 15 and 15 of 15 total anosmics, respectively (Doty et al., 1978). Both are considered as slightly pleasant (Dravnieks et al., 1984; Hummel et al., 1997). Using these odorants, we therefore attempted to minimize any brain activations that could be due to obvious differences between sensations of pleasantness/unpleasantness elicited by the odorants used as noted in other studies (Savic et al., 2002; Boyle et al., 2007b).

\section{EXPERIMENTAL PROCEDURES}

\section{Subjects}

Twenty-five healthy undergraduate students (aged 20-24 years; 19 females and six males) were included in the study. The subjects were non-smokers, right-handed, free of head colds and screened for any possible olfactory dysfunctions prior to the study. The study was reviewed and approved by the local ethics committee and declared to the National authority ( $\mathrm{N}^{\circ}$ UF: 1013; DGS 2006/0494) in accordance with the Declaration of Helsinki on biomedical studies involving human subjects. Subjects' participation also required a written informed consent and medical screening.

\section{Odor delivery}

The odors were delivered via a multi-channel custom-built olfactometer (Andrieu et al., 2011). The olfactometer was suitable for the MRI environment and generated odors with a rapid and steady on-off time $(400 \mathrm{~ms})$. The change between odorant and nonodorant conditions did not produce any thermal, tactile or auditory cues. Under baseline conditions, a constant flow of odorless, humidified air at a constant temperature was delivered to the subject through two nasal cannula nosepieces (Pro-Flow Plus ${ }^{\mathrm{TM}}$ Nasal Oral Cannula, Pro-Tech $\AA$, Murrysville, PA, USA). The use of this air as a vector embedding the odor flow prevented the detection of odor delivery by sensory systems other than chemical (such as sensitivity to changes in pressure). The pressure of each air stream (vector and odor) was controled by a flowmeter, ensuring a constant flow rate of $591 \mathrm{ml} / \mathrm{min}$ for each one. The use of solenoid valves allowed the different odorant conditions to be generated by selecting the air flow passing through encapsulated gauze pads soaked either with $7 \mu$ l of PEA or $5 \mu$ l of AA (undiluted solution: Across Organics $\AA$, Gell, Belgium). The capsules $(2 \mathrm{~cm}$ in diameter) were connected to the nosepieces by a tube which was short $(10 \mathrm{~cm})$ in order to ensure minimal adhesion and a square wave-like delivery of the odor. These supra-threshold concentra- tions were chosen following preliminary tests on a panel of five young women to obtain approximately the same self-ratings of intensity for the two odors and to ensure that both odors were constantly and correctly perceived for a sufficient amount of time corresponding to the MRI session duration. No sensation relating to trigeminal stimulation (Laska et al., 1997) was reported for PEA but a slight sensation of tickling or prickling was mentioned by four of the five subjects. The odor delivery was generated by a computer with E-Prime 2.0 (Psychology Software Tools, Pittsburg, USA) and synchronized with the onset of an inward breath by the subject (inhalation flow rate trigger). The delivery lasted $2 s$ to ensure that the odor would be smelled for the entire duration of the inhalation phase of the breathing cycle.

Before the scan session, subjects were debriefed on the purpose of the study and informed about the type of odors used. They were asked to breathe regularly through the nose only, without actively sniffing (sniffing has been shown to activate special brain processes [Sobel et al., 1998a]), and to focus on the odors without performing any others tasks. At the end of the scan, they were asked to describe their feelings about the odorants used.

\section{Experimental paradigm}

Subjects were scanned during a sequence of odorant stimulations, with either PEA or AA alternating with the non-odorant condition. Each of these odorants was delivered for either one inhalation, three successive inhalations or six successive inhalations. This procedure was repeated four times for each of the two odorants and for each of the three inhalation sequences (i.e. 1, 3, 6) corresponding to three different durations of continuous stimulation (Fig. 1). Each of these odorant stimulations (short, medium, long duration) was separated by a rest condition (odorless air flow) lasting at least $25 \mathrm{~s}$, up until the inhalation trigger. The sequences of odor type and stimulus duration were randomly determined for each subject by a computer program, which continuously monitored the breathing cycle and controled the switching device of the olfactometer.

\section{MRI data acquisition}

Magnetic resonance images were collected on a 3-T scanner (G.E. Healthcare Signa, Milwaukee, WI, USA). First of all, a highresolution T1-weighted (BRAVO FSPGR sequence) 3D anatomical scan with 134 slices, voxel size of $1 \times 1 \times 1 \mathrm{~mm}^{3}, 256 \times 256$ matrix and $256 \times 256 \mathrm{~mm}^{2}$ field of view (FOV) was recorded. Next, BOLD images were obtained covering the entire cerebrum and most of the cerebellum using an echo-planar imaging (EPI) sequence. Scan parameters included a $128 \times 128$ matrix, a repetition time (TR) of $2500 \mathrm{~ms}$, a echo time (TE) of $35 \mathrm{~ms}$ and an FOV $=256$ $\mathrm{mm}^{2}$. Thirty $4.5-\mathrm{mm}$ thick slices were acquired for each of the volumes. They were acquired in an oblique orientation $30^{\circ}$ to the anterior commissure-posterior commissure line to minimize sus-

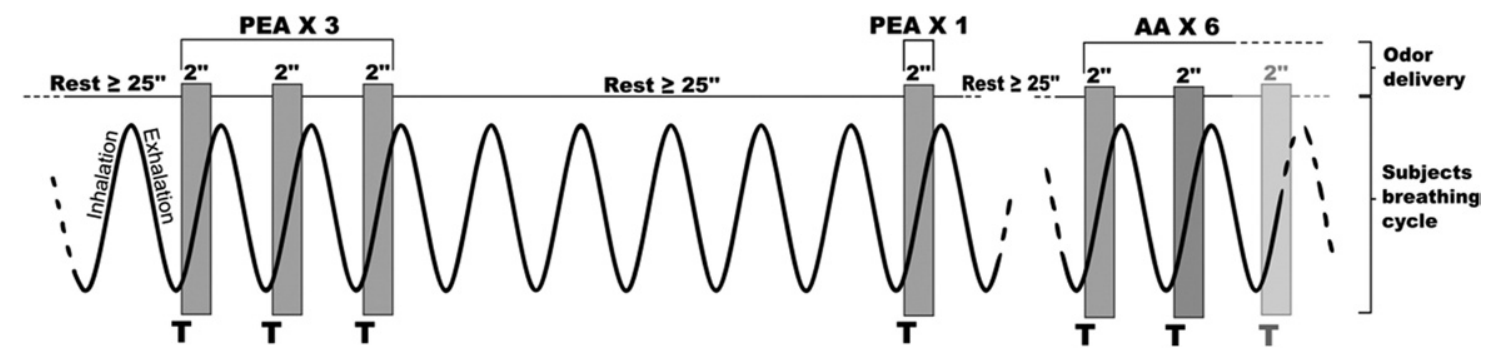

Fig. 1. Sample of the experimental procedure during the functional scan. Odorless epochs (rest) alternated with odorant stimulations (either with phenyl ethyl alcohol or isoamyl acetate: PEA and AA) synchronized with the beginning of the inward breath during one, three or six successive inhalations. This whole procedure was repeated four times in a random order during the scan. $T=$ inhalation trigger. 
ceptibility artifacts in olfactory regions of the brain: ventral portions of the temporal and frontal lobes (Gottfried et al., 2002; Sobel et al., 2003). Functional scanning was always preceded by four dummy volumes to ensure tissue steady-state magnetization. The total duration of the functional session was between 14 and 18 min, depending on the frequency of the respiratory cycle of each subject. All the scans were inserted into the matrix design for statistical analysis.

\section{Functional magnetic resonance imaging (fMRI) data analysis}

The event-related $\mathrm{fMRI}$ data were analyzed with the Brain Voyager ${ }^{\mathrm{TM}}$ QX software package (Goebel, 1996) using the general linear model (Friston et al., 1995). Functional data pre-processing included head motion correction, high-frequency filtering and spatial $(F W H M=5 \mathrm{~mm})$ and temporal Gaussian smoothing. Next, the functional data were spatially re-scaled to a resolution of $2 \times 2 \times 2$ $\mathrm{mm}^{3}$ using trilinear interpolation. Functional and anatomical images were transformed into a standard space (Talairach and Tournoux, 1988). The further analysis included two steps. Our study focused on the trigeminal properties of $A A$; these may be uncertain as they depend on the concentration used (which was selected as moderate) and are prone to variations according to individual sensitivity. We therefore initially verified the presence or absence of activations in individual analyses, subject by subject, on the trigeminal nuclei (brainstem) in order to exclude any subjects without activations. The condition of one respiratory cycle was examined to ascertain this trigeminal property of the bimodal stimulation (contrast AA-PEA). Activations $(P<0.05$, uncorrected; cluster size $>10$ voxels) in this area were observed for 17 subjects (13 females and four males). The analysis was subsequently performed in a second step on these 17 subjects only. Random effect analyses (RFX) were conducted based on statistical parameter maps from each individual subject. As the aim of our study was the time course of activation in response to the trigeminal component of an odorant, we focused the analysis only on the significant activations resulting from the bimodal stimulations minus the olfactory stimulations (AA-PEA). These activation patterns were analyzed in the three different stimulation durations corresponding to inhalations of one, three and six respiratory cycles. For the three and six respiratory cycles, we only took into account the last inspiration in order to compare similar situations, without temporal summation, but with various previous durations of odorant stimulation. Cluster activations were considered if 10 or more adjacent voxels passed the threshold of $P<0.005$ (uncorrected).

\section{RESULTS}

On the post-scan debriefing, all the subjects described the pleasantness of the odorants as neutral to positive. Of the 17 subjects, 14 reported slight sensations of tickling or prickling for the AA odorant.

Concerning the fMRI data, Table 1 gives the clusters of activations obtained with AA stimulations using PEA as a reference and according to the duration of the stimulations, that is, one, three or six successive inhalations.

During short-duration stimulations (one inhalation), activations were located in the left brainstem (trigeminal nuclei) and cerebellum. The caudate nucleus (bilaterally), the left anterior cingulate cortex, anterior insula and hippocampus were also recruited. Other cortices were virtually unaffected by activations observed exclusively in the posterior orbitofrontal cortex (Fig. 2A).

For medium-duration stimulations (the third of three successive inhalations), activations were more numerous and located predominantly in the right hemisphere. They mainly concerned the inferior, middle and superior frontal cortex and, to a lesser degree, the temporal and the occipital lobe as well as the anterior insula. Globus pallidus recruitment was observed in this contrast, as well as an activation in the right cerebellum (Fig. 2B).

During long-duration stimulations (the sixth of six successive inhalations), activations in the frontal lobe (medial part) were more restricted than in the previous situation. However activations were observed in the brainstem (pontic trigeminal tractus) and in the left post-central gyrus (SI). Activations also affected the middle cingulate cortex and the posterior insular regions. Cerebellar activations were restricted to the left hemisphere (Fig. 2C).

\section{DISCUSSION}

Previous studies comparing activations with "pure" olfactory and trigeminal stimuli have demonstrated considerable overlap in the brain areas mediating these two sensorial systems, but some structures are almost exclusively identified in cases of trigeminal stimulation (Albrecht et al., 2010). In our study, it can be assumed that there was a clear difference between the trigeminal properties of the two odorants since no subjects in the preliminary panel and no subjects tested at a post-experiment debriefing reported any specific trigeminal sensation (Laska et al., 1997) for the PEA. On the contrary, slight sensations of tickling or prickling have been reported for AA (but no sensation relative to nociception such as pain, burning, etc.) and our results identified activations in the pontic trigeminal nucleus as well as in brain regions which have been previously shown to be involved in trigeminal perception in the literature. However, one limitation of our study is that no acute behavioral assessment of the perception of the trigeminal component was performed for AA stimulation. In order to do this, a group of anosmic patients would need to be tested. It can be assumed that they would perceive an odor with $\mathrm{AA}$, used at the same concentration as in this study, thus demonstrating the trigeminal nature of this stimulation. Further research is needed in order to address these limitations of the study.

However, our results show clear differences in activation patterns according to the duration of the previous stimulation which varied from $2 \mathrm{~s}$ (one inhalation) to about $30 \mathrm{~s}$ (six successive inhalations).

When the stimulus duration was short, the results showed an activation of the caudate nucleus. This activation was no longer observed when the duration of the stimulation increased. The caudate nucleus is cited as a sub-cortical region activated by trigeminal stimulations (Hummel et al., 2009). However, it is not systematically cited in cerebral imaging studies using pure trigeminal stimuli and thus does not appear in the meta-analysis of brain imaging data by Albrecht et al. (2010). lannilli et al. (2009) mentioned the caudate nucleus as being activated using electrical or mechanical trigeminal mediating stimulations but not chemical $\left(\mathrm{CO}_{2}\right)$ stimulations. However, in subjects smelling bimodal odorants, Bengtsson et al. 
Table 1. Cerebral activations with isoamyl acetate (AA) using phenyl ethyl alcohol (PEA) as a reference and according to the duration of the stimulation (one, three and six inhalations: AA1-PEA1, AA3-PEA3, AA6-PEA6, respectively)

\begin{tabular}{|c|c|c|c|c|c|c|c|}
\hline Brain regions & & $\mathrm{x}$ & $y$ & z & $\mathrm{K}$ & Max $t$ value & $P$ value \\
\hline \multicolumn{8}{|c|}{ Contrast AA1-PEA1 } \\
\hline $\mathrm{R}$ & Caudate nucleus (head) & 6 & 0 & 3 & 58 & 3.993 & 0.001046 \\
\hline $\mathrm{R}$ & Caudate nucleus (body) & 10 & -7 & 20 & 56 & 5.76 & 0.000029 \\
\hline $\mathrm{L}$ & Caudate nucleus (body) & -11 & -6 & 18 & 44 & 3.843 & 0.001435 \\
\hline $\mathrm{L}$ & Anterior insula & -52 & 27 & 16 & 12 & 3.56 & 0.00256 \\
\hline $\mathrm{L}$ & Anterior cingulate cortex & 0 & 7 & -4 & 26 & 3.505 & 0.002932 \\
\hline $\mathrm{L}$ & Posterior orbitofrontal cortex & -25 & 14 & -9 & 58 & 4.309 & 0.00054 \\
\hline $\mathrm{L}$ & Hippocampus & -26 & -5 & -20 & 28 & 3.606 & 0.002366 \\
\hline $\mathrm{L}$ & Cerebellum: anterior lobe (culmen) & -4 & -48 & -4 & 38 & 5.273 & 0.000076 \\
\hline L & Brainstem: pontic trigeminal nucleus & -15 & -28 & -31 & 26 & 3.812 & 0.001534 \\
\hline \multicolumn{8}{|c|}{ Contrast AA3-PEA3 } \\
\hline $\mathrm{R}$ & Superior frontal gyrus & 7 & 5 & 64 & 22 & 3.503 & 0.002941 \\
\hline $\mathrm{R}$ & Superior frontal gyrus & 9 & 30 & 56 & 18 & 3.612 & 0.002341 \\
\hline $\mathrm{L}$ & Middle frontal gyrus & -36 & 9 & 48 & 116 & 4.064 & 0.000902 \\
\hline $\mathrm{R}$ & Middle frontal gyrus & 39 & -1 & 47 & 121 & 4.282 & 0.000572 \\
\hline $\mathrm{R}$ & Superior frontal gyrus & 11 & 43 & 36 & 164 & 4.257 & 0.000602 \\
\hline $\mathrm{L}$ & Middle frontal gyrus & -54 & 23 & 28 & 45 & 3.474 & 0.003132 \\
\hline $\mathrm{R}$ & Inferior frontal gyrus & 30 & 45 & 10 & 213 & 4.47 & 0.000387 \\
\hline $\mathrm{L}$ & Inferior frontal gyrus & -22 & 52 & 9 & 153 & 4.05 & 0.000929 \\
\hline $\mathrm{R}$ & Angular gyrus & 54 & -53 & 19 & 430 & 4.357 & 0.000489 \\
\hline $\mathrm{L}$ & Angular gyrus & -50 & -58 & 25 & 171 & 3.915 & 0.001235 \\
\hline $\mathrm{R}$ & Globus pallidus & 15 & -2 & 12 & 33 & 3.968 & 0.001103 \\
\hline $\mathrm{R}$ & Anterior insula & 45 & 18 & 16 & 40 & 3.596 & 0.00243 \\
\hline $\mathrm{R}$ & Middle temporal gyrus & 47 & -28 & -5 & 90 & 4.086 & 0.000862 \\
\hline $\mathrm{R}$ & Inferior temporal gyrus & 51 & -36 & -12 & 21 & 3.505 & 0.002934 \\
\hline $\mathrm{R}$ & Inferior temporal gyrus & 58 & -50 & -19 & 132 & 4.124 & 0.000796 \\
\hline $\mathrm{R}$ & Cuneus & 13 & -75 & 9 & 13 & 3.483 & 0.003068 \\
\hline $\mathrm{R}$ & Inferior occipital gyrus & 24 & -83 & -21 & 39 & 4.287 & 0.000565 \\
\hline $\mathrm{R}$ & Inferior occipital gyrus & 5 & -78 & -22 & 36 & 3.722 & 0.001855 \\
\hline $\mathrm{R}$ & Cerebellum: superior semilunar lobule & 31 & -78 & -32 & 60 & 3.946 & 0.001157 \\
\hline \multicolumn{8}{|c|}{ Contrast AA6-PEA6 } \\
\hline $\mathrm{R}$ & Superior frontal gyrus (medial part) & 5 & -12 & 56 & 321 & 5.437 & 0.000055 \\
\hline $\mathrm{L}$ & Superior frontal gyrus (medial part) & -5 & -13 & 56 & 58 & 4.021 & 0.000988 \\
\hline $\mathrm{L}$ & Middle cingulate gyrus & -4 & 1 & 46 & 244 & 4.203 & 0.000675 \\
\hline $\mathrm{L}$ & Post-central gyrus (SI) & -64 & -23 & 33 & 58 & 4.183 & 0.000703 \\
\hline L & Posterior insula & -53 & -22 & 12 & 1496 & 5.792 & 0.000028 \\
\hline $\mathrm{L}$ & Posterior insula & -49 & -12 & 12 & 44 & 3.728 & 0.001829 \\
\hline $\mathrm{R}$ & Posterior insula & 47 & -8 & 13 & 358 & 5.314 & 0.00007 \\
\hline $\mathrm{R}$ & Posterior insula & 43 & -18 & 16 & 124 & 3.927 & 0.001203 \\
\hline $\mathrm{R}$ & Superior temporal gyrus & 38 & -3 & -7 & 129 & 4.128 & 0.000788 \\
\hline $\mathrm{L}$ & Cerebellum hemisphere & -38 & -44 & -28 & 19 & 3.506 & 0.002927 \\
\hline $\mathrm{L}$ & Cerebellum: inferior semi-lunar lobule & -8 & -80 & -41 & 51 & 5.228 & 0.000083 \\
\hline $\mathrm{L}$ & Brainstem: pontic trigeminal tractus & -19 & -19 & -33 & 27 & 4.648 & 0.000268 \\
\hline
\end{tabular}

All reported activations were significant at $P<0.005$, uncorrected. Talairach coordinates are presented in $\mathrm{x}, \mathrm{y}, \mathrm{z}(\mathrm{mm})$. K is the volume of clusters (in voxels). The $t$ and $P$-values are given for the voxel showing the maximal activation. $R$ (right) and $L$ (left) refer to the brain hemisphere.

(2001) noted a widespread activation extending to the caudate nucleus in female subjects. It is possible that the caudate nucleus is only involved when a bimodal stimulus is used (which was the case in our study) but not when a pure trigeminal stimulus is applied, as an olfactory stimulus may enhance responses to a simultaneous trigeminal component of the stimulation (Hummel et al., 1996, 2003). Indeed, activations of the caudate nucleus have also been observed, sometimes in response to a pure olfactory stimulus (Poellinger et al., 2001) and particularly in odor quality discrimination (Savic, 2002), so we can conclude that in any case, its recruitment is greater for the trigeminal component of an odorant. Curiously, Poellinger's study only showed activations of the caudate nucleus for short-duration stimulations, as observed in the present study. Cerebellar activations were also noted, as was the case in our study, following intranasal trigeminal stimulation (lannilli et al., 2007) in normosmic subjects. The left posterior orbitofrontal cortex was affected by the present contrast. In the literature, as the right medial orbitofrontal cortex activation strongly characterizes olfactory stimulations (Zatorre et al., 1992; Sobel et al., 2003; Gottfried et al., 2006), trigeminal stimulations have been found in different locations within the frontal cortex, particularly in the dorsolateral orbitofrontal cortex (Hummel et al., 2005), and in the left posterior orbital gyrus alongside other 

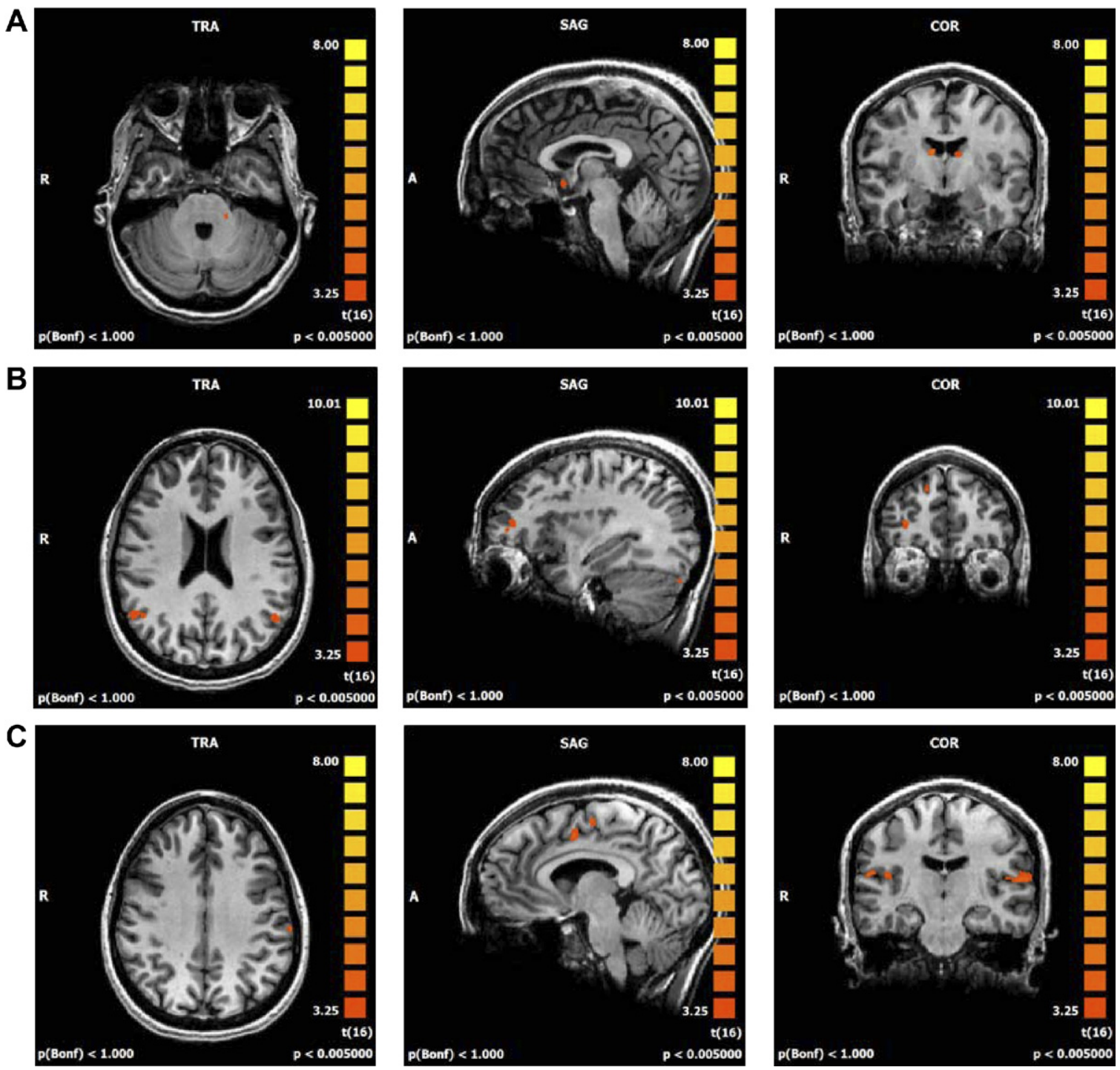

Fig. 2. Contrasting bimodal odorant (isoamyl acetate) with olfactory odorant (phenyl ethyl alcohol) indicates different patterns of activation according to the duration of the stimulation. (A) Images on the top (one inhalation). TRA: left trigeminal nuclei; SAG: left anterior cingulate cortex; COR: caudate nuclei. (B) Images on the center (three inhalations). TRA: angular gyri; SAG: right inferior frontal gurus; COR: right inferior and superior frontal gyri. (C) Images on the bottom (six inhalations). TRA: left post-central gyrus (SI); SAG: left middle cingulate and superior frontal gyri; COR: posterior insular cortices. For interpretation of the references to color in this figure legend, the reader is referred to the Web version of this article.

activations in the middle, superior and medial gyri (lannilli et al., 2007; Albrecht et al., 2010). The orbitofrontal cortex is not a homogeneous region and sub-regions can be affected by olfactory hedonics (Sobel et al., 2003) as well as by cross-modal chemosensory integration processes (Gottfried et al., 2006). Furthermore, as the right orbitofrontal cortex is mainly affected by odor stimulation, its left counterpart could be involved predominantly in trigeminal stimulation as the asymmetry shown in our results is consistent with other pools of data (Albrecht et al., 2010).

For medium-duration stimulations, our results showed a maximum number of activated brain regions in the frontal cortices and some in the temporal and occipital cortices. The cerebellum was also involved with activation restricted to the right hemisphere. The recent review by Albrecht et al. (2010) of functional imaging data identifies the cerebellum, and the frontal and temporal lobes as regions where activations due to trigeminal stimulations surpass those due to olfactory stimulations. This review is mainly based on studies using puffs of $\mathrm{CO}_{2}$ as trigeminal stimulations and we observed that, with the trigeminal component of the bimodal odor used in our study, this type of activation pattern is in someway time delayed. As Albrecht et al. (2010) mentioned, the middle frontal and temporal gyri are association cortices and they could play a role in more highly integrated steps of chemosensory perception, but the reason why they are more affected by trigeminal stimulation than by olfactory stimulation has yet to be explored. In the same way, trigeminal activations are noticeably more pronounced compared to olfactory activations (Hummel et al., 2005). 
During long-duration stimulations, one of the most striking results was the activation of the insula, predominantly in the left hemisphere. Insula activations are almost systematically noted in studies using bimodal or pure trigeminal stimuli. They have been explained by discrimination processes (Bengtsson et al., 2001), nociception (Savic et al., 2002) and by emotional processes specific to trigeminal perception (Albrecht et al., 2010). Our study suggests that these emotional processes involving the insula only emerge following a relatively long period of stimulation. Interestingly, middle cingulate activations were only seen in our study during long-duration stimulations. Middle cingulate activations with trigeminal stimulation were noted by Boyle et al. (2007b), Albrecht et al. (2009). Furthermore, it has been demonstrated that coding intensity of a pure trigeminal stimulus $\left(\mathrm{CO}_{2}\right)$ involves subregions (including the medial part) of the cingulate cortex (Bensafi et al., 2008). Therefore a sustained stimulation with a moderate intensity could have the same result on the brain activation as a stimulation with a high intensity. Furthermore, our results only showed the recruitment of the SI for these long-duration stimulations. This primary somatosensory cortex activation has been observed by Bensafi et al. (2008), Boyle et al. (2007b) and Savic et al. (2002) in response to trigeminal stimulation. Our results could suggest that somatosensitivity to the trigeminal component of a stimulation increases with its duration. Indeed, the present results are also consistent with data on brain activations related to stimulations of $\mathrm{C}$ and $\mathrm{A} \delta$ nociceptors. These two types of fiber system participate in the afferent innervation of the nasal respiratory epithelium by the trigeminal nerve. C-fibers (unmyelinated) are preferentially involved in burning sensations and $A \delta$-fibers (myelinated) in stinging and pricking sensations (Brand, 2006). Stimulations of each or both elicit activations in the posterior insula (bilaterally), in the mid-anterior cingulate cortex and in the SI (Forss et al., 2005; Ruehle et al., 2006; Staud et al., 2007; Veldhuijzen et al., 2009). These are the most consistently activated regions in pain imaging studies (Peyron et al., 2001). Although there was no painful sensation reported by the subjects in our study, these areas were recruited but only for long-duration stimulations. It is worth noting that somatosensory cortex activations (besides the middle cingulate cortex and bilateral insula) have been previously demonstrated for stimulation of the nasal mucosa with nicotine without any reports of painful sensations by the subjects (Albrecht et al., 2009).

Subregions of the cerebellum were involved regardless of the stimulus duration. This deserves special attention. Activations in the cerebellum are regularly observed in response to olfactory or trigeminal stimulations and they are usually interpreted as a result of motor control of sniffing, even in the case of passive stimulation (Sobel et al., 1998b, 2003). In our study, the activated cerebellar regions were different in the short-, medium- and longduration stimulations. This result may indicate roles of the cerebellum other than just the control of sniffing, such as a contribution to the cognitive processes linked to the per- ception of an odorant, as has previously been suggested (Qureshy et al., 2000).

\section{CONCLUSION}

This study helps to define the brain networks involved in trigeminal perception using different types of stimulation (neutral to slightly pleasant odors) and paradigm, and thus evaluates certain previous findings. Due to the differences between the two odors used ("rose" compared to "banana"), we cannot completely rule out the possibility of brain activations elicited by evocative properties specific to one or the other (i.e. not exclusively due to the trigeminal differential). However, we can assume that these possible activations would be idiosyncratic rather than univocal and therefore minimized when compared to the activations resulting from the trigeminal variation between the two odorants. Therefore, our study shows that, as is the case for olfactory perception, the time course of trigeminal perception successively involves different cortical and subcortical areas, demonstrating that the integration of this sensorial cue is just as sophisticated as brain processes linked to olfaction.

Acknowledgments-We would like to thank the Conseil Régional de Franche-Comté for its financial support and the Centre Hospitalier Universitaire de Besançon for authorizing this study. We are also grateful to Melanie Cole for her language assistance in the manuscript.

\section{REFERENCES}

Albrecht J, Kopietz R, Linn J, Sakar V, Anzinger A, Schreder T, Pollatos O, Bruckmann H, Kobal G, Wiesman M (2009) Activation of olfactory and trigeminal cortical areas following stimulation of the nasal mucosa with low concentration of $S(-)$-nicotine vapor-an fMRI study on chemosensory perception. Hum Brain Mapp 30:699-710.

Albrecht J, Kopietz R, Frasnelli J, Wiesmann M, Hummel T, Lundström JN (2010) The neural correlates of intranasal trigeminal function-an ALE meta-analysis of human functional brain imaging data. Brain Res Rev 62:183-196.

Andrieu P, Bonnans V, Comte A, Millot JL, Moulin T, Gharbi T (2011) Gustatory and olfactory systems for $\mathrm{fMRI}$ investigations. In: Alpine Brain Imaging Meeting, Champéry.

Bengtsson S, Berglund H, Gulyas B, Cohen E, Savic I (2001) Brain activation during odor perception in males and females. Neuroreport 12:2027-2033.

Bensafi M, lannilli E, Gerber J, Hummel T (2008) Neural coding of stimulus concentration in the human olfactory and trigeminal systems. Neuroscience 154:832-838.

Boyle JA, Frasnelli J, Gerber J, Heinke M, Hummel T (2007a) Crossmodal integration of intranasal stimuli: a functional magnetic resonance imaging study. Neuroscience 149:223-231.

Boyle JA, Heinke M, Gerber J, Frasnelli J, Hummel T (2007b) Cerebral activation to intranasal chemosensory trigeminal stimulation. Chem Senses 32:343-353.

Brand G (2006) Olfactory/trigeminal interactions in nasal chemoreception. Neurosci Biobehav Rev 30:908-917.

Doty RL, Brugger WPE, Jurs PC, Orndorff MA, Snyder PJ, Lowry LD (1978) Intranasal trigeminal stimulation from odorous volatiles: psychometric responses from anosmic and normal humans. Physiol Behav 20:175-185.

Dravnieks A, Masurat T, Lamm RA (1984) Hedonics of odors and odor descriptors. J Air Pollut Control Assoc 34:752-755. 
Forss N, Raij TT, Seppä M, Hari R (2005) Common cortical network for first and second pain. Neuroimage 24:132-142.

Friston KJ, Homes AP, Worsley KJ, Poline JP, Frith CD, Frackowiak RSJ (1995) Statistical parametric maps in functional imaging: a general linear approach. Hum Brain Mapp 2:189-210.

Goebel R (1996) Brain Voyager: a program for analysing and visualizing functional and structural magnetic resonance data sets. Neuroimage 3:604

Gottfried JA, Deichmann R, Winston JS, Dolan RJ (2002) Functional heterogeneity of human olfactory cortex: an event-related functional magnetic resonance imaging study. J Neurosci 15:1081910828.

Gottfried JA, Small DM, Zald DH (2006) The chemical senses. In: The orbitofrontal cortex (Zald DH, Rauch SL, eds), pp 125-172. Oxford: Oxford University Press.

Hummel T (2000) Assessment of intranasal trigeminal function. Int J Psychophysiol 36:147-155.

Hummel T, Barz S, Lotsch J, Roscher S, Kettenmann B, Kobal G (1996) Loss of olfactory function leads to a decrease of trigeminal sensitivity. Chem Senses 21:75-79.

Hummel T, Sekinger B, Wolf SR, Pauli E, Kobal G (1997) "Sniffing" sticks: olfactory performance assessed by the combined testing of odor identification, odor discrimination and olfactory threshold. Chem Senses 22:39-52.

Hummel T, Futschik T, Frasnelli J, Huttenbrink KB (2003) Effects of olfactory function, age and gender on trigeminally mediated sensations: a study based on the lateralization of chemosensory stimuli. Toxicol Lett 140-141:273-280.

Hummel T, Doty RL, Yousem DM (2005) Functional MRI of intranasal chemosensory trigeminal activation. Chem Senses 30:i205-i206.

Hummel T, Iannilli E, Frasnelli J, Boyle J, Gerber J (2009) Central processing of trigeminal activation in humans. Ann N Y Acad Sci 1170:190-195.

lannilli E, Gerber J, Frasnelli J, Hummel T (2007) Intranasal trigeminal function in subjects with and without an intact sense of smell. Brain Res 1139:235-244.

lannilli E, Del Gratta C, Gerber JC, Romani GL, Hummel T (2009) Trigeminal activation using chemical, electrical and mechanical stimuli. Pain 139:376-388.

Laska M, Distel H, Hudson R (1997) Trigeminal perception of odorant quality in congenitally anosmic subjects. Chem Senses 22:447456.

Lombion S, Comte A, Tatu L, Brand G, Moulin T, Millot JL (2009) Patterns of cerebral activation during olfactory and trigeminal stimulations. Hum Brain Mapp 30:821-828.

Peyron R, Laurent B, Garcia-Larrea L (2001) Functional imaging of brain responses to pain: a review and meta-analysis. Neurophysiol Clin 30:263-288.

Poellinger A, Thomas R, Lio P, Lee A, Makris N, Rosen BR, Kwong KK (2001) Activation and habituation in olfaction-an fMRI study. Neuroimage 13:547-560.
Qureshy A, Kawashima R, Imaran M, Sugiura M, Goto R, Okada K, Inoue K, Itoh M, Shormann T, Zilles K, Fukuda H (2000) Functional mapping of human brain in olfactory processing: a PET study. J Neurophysiol 84:1656-1666.

Royet JP, Hudry J, Zald DH, Godinot D, Gregoire MC, Lavenne F, Costes N, Holley A (2001) Functional neuroanatomy of different olfactory judgments. Neuroimage 13:506-519.

Royet JP, Plailly J, Delon-Martin C, Kareken DA, Segebarth C (2003) fMRI of emotional responses to odors: influence of hedonic valence and judgment, handedness and gender. Neuroimage 20: 713-728.

Ruehle BS, Handwerker HO, Lennerz JKM, Ringler R, Forster C (2006) Brain activation during input from mechanoinsensitive versus polymodal C-nociceptors. J Neurosci 26:5492-5499.

Savic I (2002) Brain imaging studies of the functional organization of human olfaction. Neuroscientist 8:204-211.

Savic I, Gulyas B, Berglund H (2002) Odorant differentiated pattern of cerebral activation: comparison of acetone and vanillin. Hum Brain Mapp 17:17-27.

Sobel N, Prabhakaran V, Desmond JE, Glover GH, Goode RL, Sullivan EV, Gabrieli JDE (1998a) Sniffing and smelling: separate subsystems in the human olfactory cortex. Nature 392:282-286.

Sobel N, Prabhakaran V, Hartley CA, Desmond JE, Zhao Z, Glover GH, Gabrieli JDE, Sullivan EV (1998b) Odorant-induced and sniffinduced activation in the cerebellum of the human. $J$ Neurosci 18:8990-9001.

Sobel N, Prabhakaran V, Zhao Z, Desmond JE, Glover GH, Sullivan EV, Gabrieli JDE (2000) Time course of odorant-induced activation in the human primary olfactory cortex. J Neurophysiol 83:537-551.

Sobel N, Johnson BN, Mainland J, Yousem D (2003) Functional neuroimaging of human olfaction. In: Handbook of olfaction and gustation, 2nd ed (Doty RL, ed), pp 251-273. New York: Dekker.

Staud R, Craggs JG, Robinson ME, Perlstein WM, Price DD (2007) Brain activity related to temporal summation of C-fiber evoked pain. Pain 129:130-142.

Talairach J, Tournoux P (1988) Co-planar stereotaxic atlas of the human brain. 3-dimensional proportional system: an approach to cerebral imaging. New York: Thieme Medical.

Veldhuijzen DS, Nemenov MI, Keaser M, Zhuo J, Gullapalli RP, Greenspan JD (2009) Differential brain activation associated with laser-evoked burning and prickling pain: an event-related fMRI study. Pain 141:104-113.

Yousem DM, Williams SCR, Howard RO, Andrew C, Simmons A, Allin M, Geckle RJ, Suskind D, Bullmore ET, Brammer MJ, Doty RL (1997) Functional MR imaging during odor stimulation: preliminary data. Radiology 204:833-838.

Zatorre RJ, Jones-Gotman M, Evans AC, Meyer E (1992) Functional localization and lateralization of human olfactory cortex. Nature 360:339-340. 